\title{
Phonon Transport in Strong-Scattering Media
}

\author{
Ping Sheng, Minyao Zhou, and Zhao-Qing Zhang \\ Exxon Research and Engineering Company, Route 22 East, Annandale, New Jersey 08801
}

(Received 1 June 1993)

\begin{abstract}
We study phonon transport in random 3D lattices when the mean free path $/$ becomes comparable to the lattice spacing $a$. Through numerical simulation, it is shown that in contrast to the electronic case, here the Ioffe-Regel criterion is inaccurate in the prediction of phonon localization. By using the Thouless criterion to pinpoint the mobility edge $\omega_{c}$, a broad frequency regime is found below $\omega_{c}$ where $k l \leq 1$ but the phonon transport is still diffusive. The diffusion constant in this regime is given quantitatively by $v_{E} a / 3$, where $v_{E}$ is an energy transport velocity derived from a continuity relation.
\end{abstract}

PACS numbers: 66.30.Dn, 05.60. $+\mathrm{w}, 63.50 .+\mathrm{x}, 66.70 .+\mathrm{f}$

Phonon transport is responsible for heat conduction in electrically insulating solids. Over the past few decades, there has been widespread interest in relating the "plateau" phenomenon, universally observed in the temperature dependence of heat conductivity $\kappa(T)$ in disordered materials, to the generic phonon transport behavior in strong-scattering media [1-9]. Here the most intriguing issue is: What happens when the phonon mean free path $l$ becomes comparable to the lattice constant $a$, and the product $k l$, where $k$ is the phonon wave vector, is less than or equal to one? On the one hand, the application of the Ioffe-Regel criterion [10], that $k l \leq 1$ implies localization, would preclude the further rise in $\kappa(T)$ that is generally observed to follow the plateau at temperatures $\gtrsim 30 \mathrm{~K}$. On the other hand, if the diffusive behavior persists with $k l \leq 1$, which would allow consistency with the observed $\kappa(T)$ data [11], then what should be the formula for the diffusion constant $D$, now that the weakscattering assumption underlying the classical kinetic formula for $D$ is no longer valid? Also, what should be the implication for the Ioffe-Regel criterion in that case? Up to now, while there are theories addressing these two aspects of the issue separately $[1,3,7,9,12]$, there has yet been no clear resolution of the two conflicting viewpoints based on a single structural model, such that both aspects of the problem may be treated on a unified basis.

In this Letter, we report the results of direct numerical simulation of phonon transport on a model system, consisting of a 3D simple cubic lattice with a significant fraction of randomly located missing sites, but which is still far from the percolation threshold. It is shown that in contrast to the electronic case, here the Ioffe-Regel criterion is inaccurate in the prediction of phonon localization. This is due to the existence of a broad frequency regime below the mobility edge where $k l \leq 1$ but the phonon transport is still diffusive in character. The fact that the phonons are not localized in this strong-scattering regime is supported by an independent calculation of the Edwards and Thouless criterion [13], which also provides the means of pinpointing the mobility edge. The phonon diffusion constant in the regime of $k l \leq 1$ is found to be quantitatively described by $v_{E} a / 3$, where $v_{E}$ is an energy velocity calculated by following a recent proposal by the Amsterdam group [14], i.e., as the ratio between an "energy current" and the local energy, where the two quantities satisfy a continuity relation. Similarity and differences with the Einstein picture [1] of incoherent phonon transport are noted below.

Consider a $79 \times 79 \times 79$ simple cubic lattice with a site occupation probability $p=0.65$. Since the 3D site percolation threshold is $p_{c} \simeq 0.31$, the lattice is far from the threshold condition, and the correlation length is 1-2 lattice constants. The choice of $p=0.65$ is based on the earlier work [11] which showed a value of $p=0.55$ to 0.65 would yield a $\kappa(T)$ that is consistent with the observed plateau phenomenon in amorphous solids. Vibrational motion of the lattice is governed by the equation of motion

$$
m \frac{d^{2} u_{\alpha}}{d t^{2}}=K \sum_{[\beta]} f_{\alpha \beta}\left(u_{\beta}-u_{\alpha}\right)+S_{\alpha}(t),
$$

where $u_{a}$ denotes the scalar displacement of the particle on site $\alpha$, the summation $[\beta]$ is over the nearest neighbors of the $\alpha$ site, $f_{\alpha \beta}=1$ if $\alpha$ and $\beta$ are both occupied, and $f_{\alpha \beta}=0$ otherwise, $S_{\alpha}(t)$ is a source term, $m$ is the mass of the particle on each occupied site, and $K$ is the spring constant.

The phonon mean free path $l$ is generally defined as the decay length of the configurationally averaged Green's function, i.e.,

$$
\begin{aligned}
& \langle G(\mathbf{r})\rangle \sim \exp (i k r) / r, \\
& l=\frac{1}{2}(\operatorname{Im} k)^{-1} .
\end{aligned}
$$

Here the Green's function represents the classical response to a point harmonic force, which is equivalent to letting $S_{\alpha}(t)=\exp (i \omega t) \delta_{\alpha, 0}$ in Eq. (1). It is easy to show that in the case of a planar source, $l$ defines the decay length of configurationally averaged response perpendicular to the plane. By placing $S_{\alpha}(t)$ on one surface of the sample, Eq. (1) is integrated forward in time until the disturbance reaches the opposite surface of the sample. The integration is then stopped, and two types of averages are carried out on the resulting $u_{\alpha}$ spatial pattern. 
The first is to average $u_{a}$ for every plane parallel to the source plane, and to plot the result as a function of distance from the source plane. Exceedingly good exponentials are obtained and the decay length yields $l$ directly. In all cases, $l \ll$ sample size $L$. The second is to perform a fast Fourier transform on the spatial $u_{\alpha}$ pattern, and to plot the result as a function of $|\mathbf{k}|$. For a given frequency $\omega$, the Fourier amplitude is a peaked function of $k$. If the peak position is denoted by $k_{0}$, then it is found that the relation $k_{0} \cong \omega / v$ is followed rather well, where $v$ is the low-frequency acoustic velocity, $\approx 0.64 a \omega_{0}$, where $\omega_{0}$ $=\sqrt{K / m}$. However, the width of the amplitude distribution function increases with $\omega$, so the concept of wave vector becomes increasingly ill-defined at higher frequencies. Nevertheless, if we let $k_{0}$ play the role of "phonon wave vector," then the variation of $k_{0} l$ as a function of $\omega$ is shown in Fig. 1. It is seen that $k_{0} l$ is slightly less than 1 for $\omega \gtrsim 2.2 \omega_{0}$. Here the phonon band edge is noted to be at $\omega=3.1 \omega_{0}$. In fact, $l$ becomes less than $a$ at $\omega \cong 1.35 \omega_{0}$, and continues to decrease as $\omega$ increases. (It should be noted that the transport mean free path $l^{*}$, defined from the Boltzmann transport equation, cannot be less than $a$; but $l$, which represents the decay rate of the coherent phase, can be less than a.)

The Ioffe-Regel criterion [10] states that the plane wave description cannot be applicable when $k_{0} l \leq 1$. Mott and Davis [15] have given the Ioffe-Regel criterion a stronger interpretation that $k_{0} l \leq 1$ implies localization. In the present case, a strict application of the latter interpretation would place the mobility edge at $\omega \sim 2.2 \omega_{0}$. However, if the criterion is interpreted loosely to mean that $k_{0} l \lesssim$ some constant $\approx 1$ implies localization, then the mobility edge can be placed anywhere between $2.2 \omega_{0}$ and 3.1 $\omega_{0}$. Hence the Ioffe-Regel criterion is inherently inaccurate for the prediction of phonon localization. In

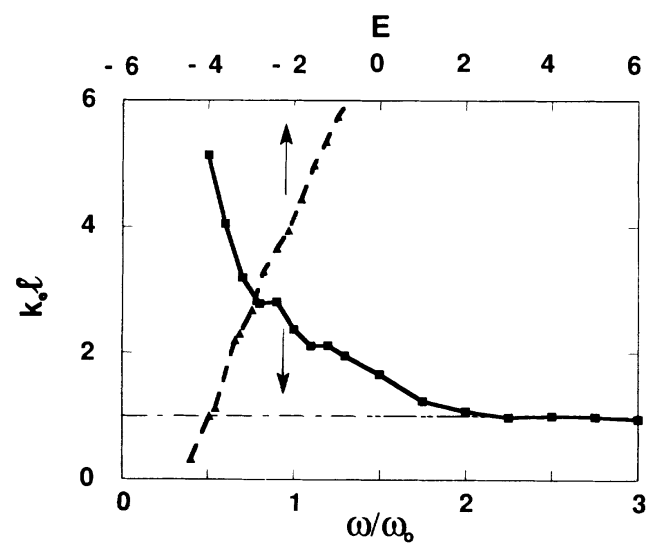

FIG. 1. Product $k_{0} l$ plotted as a function of frequency. Solid line denotes the phonon case. Dashed line denotes the electronic case. Finite-size scaling [17] gives the electronic mobility edge at $\sim-4.3$ (upper scale) in units of the transfer matrix element. The curve for $E>0$ can be inferred from the electron-hole symmetry. contrast, for the electronic case a calculation of $k l$ on the same lattice structure yields the dashed line [16] in Fig. 1 , which crosses the line $k_{0} l=1$ with a reasonably steep slope, and is thus relatively accurate in its prediction of the mobility edge as can be verified through independent finite-size scaling calculations [17]. This striking difference in the frequency dependence of $k_{0} l$ is due to the different dispersion relations of phonon and electron. Since a phonon has the classical linear dispersion at low frequencies, its plane-wave scattering follows the Rayleigh frequency dependence of $l \sim \omega^{-4}$. This is why for phonons $k_{0} l$ drops steeply until $l$ reaches the order of $a$, then the Ioffe-Regel criterion dictates that some new transport characteristics must emerge. For electrons, on the other hand, scattering can be strong at small $\mathbf{k}$, which accounts for its main difference with the phonons [18].

In order to have an accurate determination of the phonon mobility edge, we resort to the Thouless criterion that the ratio $\delta E / \Delta E$ should exhibit a sharp drop to zero as $\omega$ increases across the mobility edge. Here $\delta E$ is defined as the change in the energy eigenvalue when the boundary condition on the sample is changed from symmetric to antisymmetric, and $\Delta E$ is the energy level separation. The Thouless criterion is based on the physical reasoning that for a localized state in an infinite sample, $\delta E=0$ because it cannot be sensitive to what happens at the boundary. In contrast, $\delta E / \Delta E$ can be $>1$ for delocalized wave states. This well-known criterion is the basis of the scaling theory of localizations. To implement this criterion for our system, we have numerically diagonalized the force matrix whose elements are given by the first term on the right-hand side of Eq. (1). The largest sample used is $60 \times 60 \times 60$, periodically repeated, with identical Bloch conditions applied to the three pairs of surfaces in three orthogonal directions, where the Bloch wave vector $k$ is adjustable between 0 and $\pi / L$. For a fixed frequency interval, all the eigenvalues are calculated as a function of $k$ to trace their sensitivity to the boundary condition. $\delta E$ is defined as the integral of $|d(\delta E) / d k|$ from 0 to $\pi / L$, averaged over about ten neighboring eigenvalues inside the frequency interval. $\Delta E$, on the other hand, is the average level separation at $k=0$ for the same number of eigenvalues. The ratio $\delta E / \Delta E$ is then monitored as a function of the center frequency of the interval. The results are plotted in Fig. 2 for $L=24,32$, and 60. An abrupt drop in $\delta E / \Delta E$ is seen around $\omega_{c}=2.92 \omega_{0}$ in all three cases, where above $\omega_{c}$ all the eigenvalues are observed to be essentially invariant as $k$ is changed $\left(\delta E \simeq 0\right.$ ), and below $\omega_{c}$ the quantity $\delta E$ becomes larger than $\Delta E$. The fact that $\omega_{c}$ is indeed the mobility edge is further supported by the fact that the three cases of different $L$ cross each other at $\omega_{c}$, in agreement with the scale invariance property at the mobility edge.

The identification of $\omega_{c}$ implies the existence of a strong-scattering regime, $\omega_{c}>\omega>2.2 \omega_{0}$, in which $k_{0} l$ $\leq 1$ but the states are delocalized. Instead of identifying the mobility edge, here the Ioffe-Regel criterion separates 


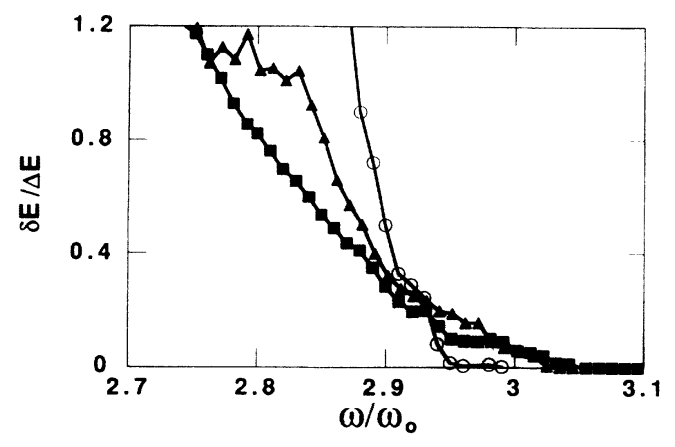

FIG. 2. $\delta E / \Delta E$ plotted as a function of frequency for a single configuration of $L=60(0), 32(\square)$, and $24(\Delta)$. The drop at $\omega_{c} \cong 2.92 \omega_{0}$ signals the mobility edge.

the weak-scattering regime from this strong-scattering regime. To examine the transport characteristics in different regimes, we employed direct simulation in the space-time domain. By letting

$$
S_{a}(t)=\exp \left[-t^{2} / t_{0}^{2}\right] \cos \omega t \delta_{a, a_{0}}
$$

in Eq. (1), with $\alpha_{0}$ chosen to be a site near the center of the $L=79$ sample, and $t_{0}=6 \pi / \omega$, the space-time evolution of this Gaussian pulse (with internal frequency $\omega$ ) is easily monitored. We calculate $\left\langle R^{2}\right\rangle$ as

$$
\left\langle R^{2}\right\rangle=\sum_{a} r_{a}^{2} u_{a}^{2}(t) / \sum_{a} u_{\alpha}^{2}(t),
$$

where $r_{\alpha}$ is the distance from site $\alpha$ to site $\alpha_{0}$. The variation of $\left\langle R^{2}\right\rangle$ as a function of time invariably exhibits an asymptotic linear behavior for all frequencies up to $\omega_{c}$. The fact that phonon transport is diffusive at low frequencies, where the scattering is weak, is well known [9]. The focus of this work, however, is on the intermediate to high frequencies, where the scattering is strong. For a particular intermediate frequency of $\omega=2.5 \omega_{0}$ this diffusive behavior is shown in the inset to Fig. 3. A phonon diffusion constant $D(\omega)$ may be defined as $\left[d\left\langle R^{2}\right\rangle\right]$ $d t] / 6$ in the asymptotic regime. The finite width of the Gaussian pulse means $D(\omega)$ represents an average over the neighboring frequencies. However, a variation of $t_{0}$ shows only minor effect on the value of $D(\omega)$. The diffusion constant evaluated from Eq. (6) is plotted with solid triangles in Fig. 3.

A quantitative description of diffusive wave transport in the regime of strong scattering requires a valid formula for the calculation of its diffusion constant. From dimensional analysis the diffusion constant has to be the product of $l^{*}$ and a velocity. Since the minimum $l^{*}$ is the lattice spacing $a$, the crucial question here is what should be taken as the transport velocity. The usual definitions of phase and group velocities require the wave vector and the dispersion relation to be well defined. Both become suspect in the strong scattering regime where $k_{0} l \leq 1$. In the case of phonons Einstein had proposed an incoherent transport velocity [13] as the product of one-half the pho-

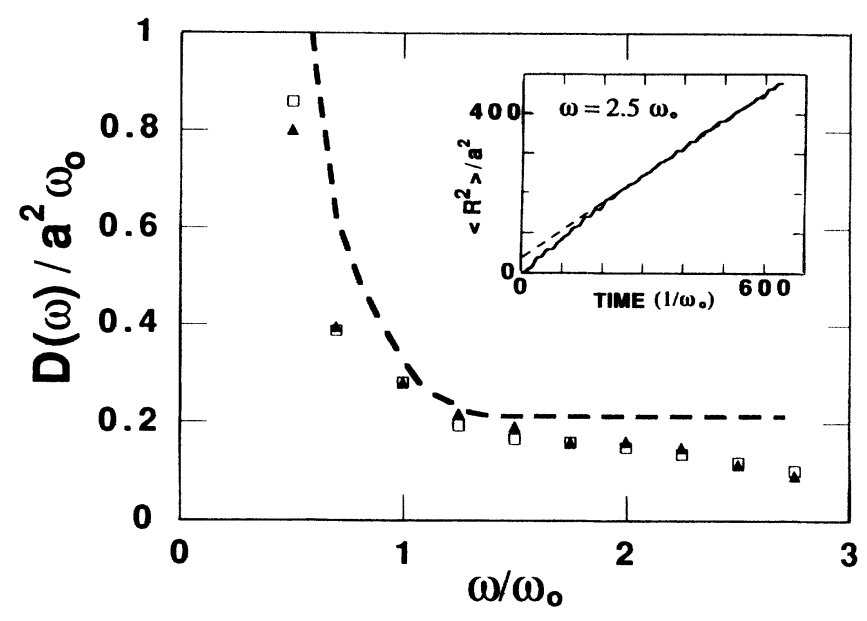

FIG. 3. Diffusion constant plotted as a function of frequency. Solid triangles denote the time-space simulation results; open squares denote the result calculated from $v_{E} l^{*} / 3$. For the inset, the time origin of the $x$ axis is defined to be the point at which the input pulse, Eq. (4), is at its maximum.

non frequency $(\omega / 2 \pi)$ (optical phonon in the original version) and $a$. However, that is seen to give an increasing trend for $D(\omega)$ as $\omega$ increases, opposite to what is obtained from the simulation results shown in Fig. 3. Recently, there was a proposal [14] which suggested that for classical waves, the transport velocity should be defined from the rate of local energy flow. Following this suggestion, we write the total energy at site $\alpha$ as

$$
E_{\alpha}=\frac{m}{2} \dot{u}_{\alpha}^{2}+\frac{K}{4} \sum_{[\beta]} f_{\alpha \beta}\left(u_{\beta}-u_{\alpha}\right)^{2},
$$

where the overdot denotes time derivative. The rate of change for $E_{\alpha}$ may be put in the form of a continuity equation. By taking the time derivative of Eq. (6) and using Eq. (1) to replace the $\ddot{u}_{\alpha}$ term, we obtain

$$
\begin{aligned}
& \frac{d E_{\alpha}}{d t}=-\sum_{[\beta]} J_{\alpha \beta}, \\
& J_{\alpha \beta}=-\frac{1}{2} f_{\alpha \beta}\left(u_{\beta}-u_{\alpha}\right)\left(\dot{u}_{\beta}+\dot{u}_{\alpha}\right) .
\end{aligned}
$$

By writing $-J_{\alpha \beta} / \frac{1}{2}\left(E_{\alpha}+E_{\beta}\right)$ as $v_{\alpha \beta}$, an energy velocity $v_{E}$ may be defined as

$$
v_{E}=\left\langle\left(\frac{1}{2} \sum_{[\beta]} v_{\alpha \beta}^{2}\right)^{1 / 2}\right\rangle_{a}
$$

where the factor $\frac{1}{2}$ accounts for the two directions along each axis, and the angular brackets denote averaging over all sites. The advantage of this definition is that it is purely local in character and does not depend on the validity of the plane wave description. $v_{E}$ can also be generalized to the weak-scattering regime, i.e., when the coherence of the wave extends over a region of $l>1$, by first performing an average on $v_{\alpha \beta}$ over a region of radius $l / 2$ centered at site $\alpha$. If that result is called $\bar{v}_{\alpha \beta}$, then a 
generalized $v_{E}$ may be obtained from Eq. (8) through the replacement of $v_{a \beta}^{2}$ by $\bar{v}_{a \beta}^{2}$. In Fig. 3 , the diffusion constant calculated from the formula

$$
D=\frac{1}{3} v_{E} l^{*},
$$

where

$$
l^{*}=\left\{\begin{array}{l}
l \text { if } l>a, \\
a \text { if } l \leq a,
\end{array}\right.
$$

is plotted as open squares. The agreement with the result obtained from space-time simulation is remarkably good, indicating the validity of Eq. (9) for both the weak- and strong-scattering regimes. In contrast, if we use the traditional formula $D=v l^{*} / 3$ derived from perturbation theory, where $v$ is the low-frequency acoustic velocity and $l^{*}$ is given by Eq. (10), then the result is indicated by the dashed line. In the strong-scattering regime the actual diffusion constant is seen to be significantly lower, percentage-wise, than what is anticipated from this traditional formula.

From the derivation of $v_{E}$, it is seen that the essential difference with the Einstein model is that in the present case the physical picture always involves nearest-neighbor pairs, and the remnant local phase coherence between the pairs, while imperfect, still plays a significant role in determining $v_{E}$ and its frequency dependence. A simplified effective medium theory for the calculation of $v_{E}$ is presently being considered.

In the context of heat conductivity, our result supports the picture which ascribes the plateau phenomenon to Rayleigh scattering plus the relaxational scattering by two-level systems, and the subsequent rise in $\kappa(T)$ to the contribution of strongly scattering phonons, whose frequencies also coincide with the peak in the phonon density of states. As a result, at room temperatures the dominant contribution to $\kappa(T)$ is due to the diffusive transport of these phonons. Also, since phonon is just one type of classical wave, the present results could have more general implications. In particular, light in 3D random media is speculated to have a similar diffusive regime pri- or to reaching its mobility edge, if one exists.

[1] A. Einstein, Ann. Phys. (N.Y.) 35, 679 (1911).

[2] R. C. Zeller and R. O. Pohl, Phys. Rev. B 4, 2029 (1971).

[3] D. G. Cahill and R. O. Pohl, Solid State Commun. 70, 927 (1989).

[4] P. B. Allen and J. L. Feldman, Phys. Rev. Lett. 62, 645 (1989).

[5] C. C. Yu and J. J. Freeman, Phys. Rev. B 36, 7620 (1987).

[6] J. E. Graebner, B. Golding, and L. C. Allen, Phys. Rev. B 34, 5696 (1986).

[7] S. Alexander, O. Entin-Wohlman, and R. Orbach, Phys. Rev. B 34, 2726 (1986)

[8] E. Akkermans and R. Maynard, Phys. Rev. B 32, 7850 (1985).

[9] G. A. Slack, in Solid State Physics, edited by F. Seitz and D. Turnbull (Academic, New York, 1979), Vol. 34, p. 1.

[10] A. F. Ioffe and A. R. Regel, Prog. Semicond. 4, 237 (1960).

[11] P. Sheng and M. Y. Zhou, Science 253, 539 (1991).

[12] C. A. Condat and T. R. Kirkpatrick, in Scattering and Localization of Classical Waves in Random Media, edited by P. Sheng (World Scientific, Singapore, 1990), p. 423.

[13] J. T. Edwards and D. J. Thouless, J. Phys. C 5, 807 (1972).

[14] M. P. van Albada, B. A. van Tiggelen, A. Lagendijk, and A. Tip, Phys. Rev. Lett. 66, 3132 (1991).

[15] See, for example, N. F. Mott and E. A. Davis, Electronic Processes in Non-crystalline Materials (Oxford Univ. Press, Oxford, 1971), p. 3.

[16] The calculation of $l$ is done by using the formula $3 D / v_{t}$, where $D$ is simulated numerically and $v_{t}$ is calculated in the coherent potential approximation with the tight binding model where the energy of the occupied sites is zero and that of the unoccupied site is $\infty$.

[17] C. M. Soukoulis, E. N. Economou, and G. Grest, Phys. Rev. B 36, 8649 (1987).

[18] This difference has been noted by J. K. Flicker and P. L. Leath, Phys. Rev. B 7, 2296 (1973). 